\title{
Prevalence of Multiple-Level Spondylolysis and the Bone Union Rates among Growth-Stage Children with Lower Back Pain
}

\author{
Noriyuki Iesato $^{1)}$, Kousuke Iba ${ }^{1)}$, Mitsunori Yoshimoto' ${ }^{1)}$, Hidenori Otsubo ${ }^{2)}$, Tomoaki Kamiya ${ }^{1)}$, Tsuyoshi Miyakawa ${ }^{3)}$ \\ Yuko Narita ${ }^{3)}$, Makoto Emori ${ }^{1)}$, Atsushi Teramoto ${ }^{1)}$ and Toshihiko Yamashita ${ }^{1)}$ \\ 1) Department of Orthopaedic Surgery, Sapporo Medical University School of Medicine, Sapporo, Japan \\ 2) Sapporo Sports Clinic, Sapporo, Japan \\ 3) Sports Medical Center, Obihiro Kyokai Hospital, Obihiro, Japan
}

\begin{abstract}
:
Introduction: Lumbar spondylolysis is usually single level, and only a few multiple-level cases have been reported. We investigated the frequency of multiple-level spondylolysis and the bone union rates among growth-stage children with lower back pain (LBP).

Methods: The subjects were growth-stage children examined for LBP between April 2013 and December 2018. All patients with LBP persisting for at least 2 weeks and severe enough to make playing sports difficult underwent lumbar plain radiogram, computed tomography, and magnetic resonance imaging. The cases diagnosed as multiple-level spondylolysis and classified as early or progressive stage received conservative treatment to achieve bone union.

Results: A total of 782 growth-stage children were examined for LBP. Of them, 243 children (31.1\%) were diagnosed with lumbar spondylolysis. Of these 243 children, 23 (9.5\%) children had multiple-level spondylolysis. Of the children diagnosed with multiple-level spondylolysis, most children $(87.0 \%)$ had pars defects in the early or progressive stage in which bone union could be expected. Most children (78.3\%) had pars defects in the terminal stage and combined with these defects, had pars defects in the early or progressive stage at a different spinal level.

Twenty children diagnosed with multiple-level spondylolysis who also had pars defects in the early or progressive stage received conservative treatment for bone union, which was achieved in 31 of 39 sites $(79.5 \%)$. The bone union rate by stage was $92.9 \%$ (26 of 28 sites) in the early stage and $45.5 \%$ (5 of 11 sites) in the progressive stage.

Conclusions: In cases of multiple-level spondylolysis, bone union is likely to be achieved with conservative treatment when the pars defects are in the early or progressive stage. Therefore, the first choice of treatment should be conservative treatment to achieve bone union, the same for single-level spondylolysis.
\end{abstract}

Keywords:

Multiple-level spondylolysis, Prevalence, Bone union rate

Spine Surg Relat Res 2021; 5(4): 292-297 dx.doi.org/10.22603/ssrr.2020-0165

\section{Introduction}

The prevalence of lumbar spondylolysis is reported to be $6 \%-8 \%{ }^{1,2}$. In most cases, it occurs at a single level of the vertebra; there are only a few reports of multiple-level spondylolysis $^{2-4)}$. Sakai et al. $^{2)}$ reported that the prevalence of lumbar spondylolysis with pars defects in the terminal stage among Japanese adults over 20 years old was 5.9\%. Most of the cases in this study were single-level spondylolysis, with only $0.25 \%$ prevalence of multiple-level spondylolysis. They reported that multiple-level spondylolysis made up $4.3 \%$ of all spondylolysis cases.

The etiology of lumbar spondylolysis involves a stress fracture of the pars interarticularis of the vertebral arch in the lumbar spine during growth stage, which results in pseudarthrosis if bone union was not achieved. Bone union is more likely to be achieved if the diagnosis is made and treatment is started early; thus, early diagnosis is particularly important. Lumbar magnetic resonance imaging (MRI) of bone marrow edema of the adjacent vertebral pedicle is use- 


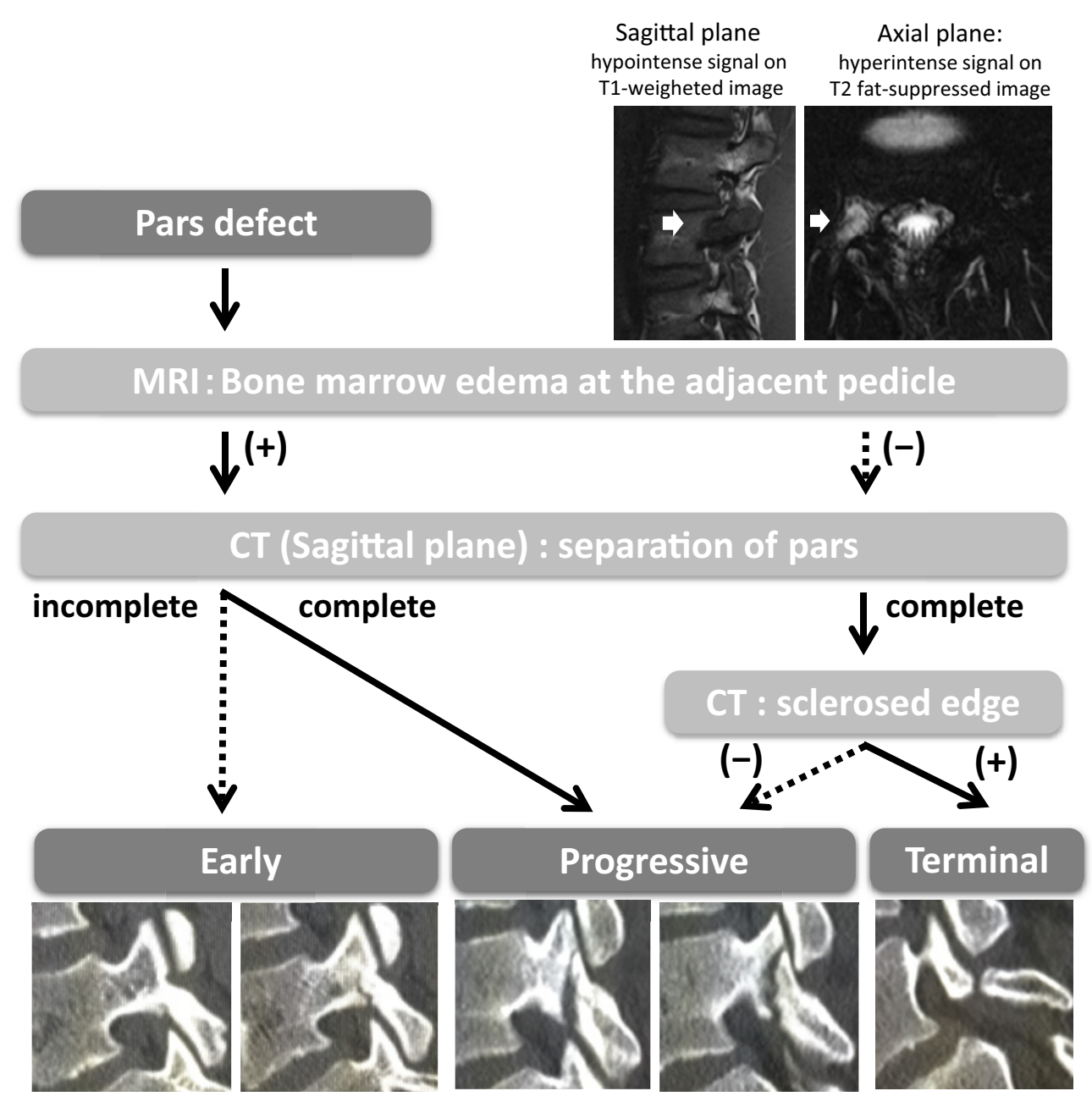

Figure 1. Stage classification of lumbar spondylolysis by magnetic resonance imaging (MRI) and computed tomography (CT).

ful for early diagnosis ${ }^{5}$. Its presence indicates that the condition is in the initial phase of onset and bone union can be expected. Its absence indicates that the condition is in the late phase of onset, and bone union cannot be expected.

There have been no previous reports on bone union rates with conservative treatment for multiple-level spondylolysis. In this study, we investigated the prevalence of multiplelevel spondylolysis and the bone union rate among growthstage children with lower back pain (LBP).

\section{Materials and Methods}

The subjects were children (growth stage, 6-18 years old) examined for LBP at the Obihiro Kyokai Hospital Sports Medical Center between April 2013 and December 2018 and diagnosed with lumbar spondylolysis.

All patients with LBP persisting for at least 2 weeks and severe enough to make playing sports difficult underwent lumbar plain radiograms, lumbar computed tomography (CT), and lumbar MRI. The presence of pars defects was assessed using lumbar CT by reconstructing sagittal images and oblique axial images aligned with the inclination of the vertebral $\operatorname{arch}^{6}$. With lumbar MRI, in addition to regular axial images around the intervertebral disc level and sagittal images (T1-weighted imaging: T1 and T2-weighted imaging: $\mathrm{T} 2$ ), axial images at the vertebral pedicle level (T1, T2, T2 fat-suppressed imaging: T2FS) and coronal images at the vertebral pedicle (T1) were recorded. Lumbar spondylolysis was diagnosed using these images and categorized according to the disease stage based on a modification of the categorizations used by Fujii and Sairyo et al. ${ }^{7,8}$. First, when the pars defect was observed in plain radiograms or CT images, we checked whether the bone marrow edema in the vertebral pedicles exhibited hypointense signal on T1 and hyperintense signal on $\mathrm{T} 2$ and $\mathrm{T} 2 \mathrm{FS}^{5}$. Among the pars defects exhibiting bone marrow edema in the adjacent vertebral pedicle, the defects limited to the ventral side of the vertebral arch and bony continuity on the dorsal side in CT sagittal images were considered in the early stage, and the defects extended to the dorsal side and with complete separation of the vertebral arch were considered in the progressive stage. Among the pars defects without bone marrow edema in the pedicle, the defects were confirmed that the separation has reached the dorsal side of the vertebral arch (complete separation) and checked the edge of the separation has sclerosed or not in CT sagittal images. In CT images, sclerosed and not sclerosed edges were considered in the terminal and progressive stages, respectively (Fig. 1). 
Table 1. All Cases of Multiple-Level Lumbar Spondylolysis.

\begin{tabular}{|c|c|c|c|c|c|c|c|c|c|c|c|c|c|}
\hline \multirow[t]{2}{*}{ No. } & \multirow[t]{2}{*}{ Sex } & \multirow[t]{2}{*}{ Age } & \multirow[t]{2}{*}{ Sports } & \multirow[t]{2}{*}{$\begin{array}{l}\text { Spinal } \\
\text { levels }\end{array}$} & \multirow{2}{*}{\multicolumn{2}{|c|}{$\begin{array}{l}\text { Number } \\
\text { of pars } \\
\text { defects }\end{array}$}} & \multicolumn{5}{|c|}{ Stage classification (spinal level, R/L) } & \multirow{2}{*}{$\begin{array}{c}\text { Bony union } \\
\text { : Union } \\
\times: \text { Pseudarthrosis } \\
\text { (Spinal level: } R / L \text { ) }\end{array}$} & \multirow{2}{*}{$\begin{array}{l}\text { Bony union } \\
\text { judgment } \\
\text { period } \\
\text { (days) }\end{array}$} \\
\hline & & & & & & & Most cranial s & side & Middle side & Mos & st caudal side & & \\
\hline 1 & $\mathrm{~F}$ & 16 & Tennis & $\mathrm{L} 2+\mathrm{L} 5$ & 3 & $\mathrm{~L} 2$ & -/terminal & & & L5 & Terminal/terminal & & \\
\hline 2 & M & 17 & Soccer & L4+L5 & 3 & L4 & Early/- & & & L5 & Terminal/terminal & L4: $\bigcirc /-$ & 77 \\
\hline 3 & $\mathrm{~F}$ & 16 & Rugby & $\mathrm{L} 2+\mathrm{L} 3+\mathrm{L} 4$ & 6 & $\mathrm{~L} 2$ & $\begin{array}{l}\text { Progressive/ } \\
\text { progressive }\end{array}$ & L3 & Early/early & L4 & Terminal/terminal & $\begin{array}{c}\text { L2: } \bigcirc / \bigcirc, \\
\text { L3: } \bigcirc / \bigcirc\end{array}$ & 157 \\
\hline 4 & $\mathrm{~F}$ & 13 & Tennis & L4+L5 & 4 & L4 & Early/early & & & L5 & Terminal/terminal & L4: $\bigcirc / \bigcirc$ & 89 \\
\hline 5 & M & 14 & Soccer & L4+L5 & 4 & L4 & $\begin{array}{l}\text { Progressive/ } \\
\text { progressive }\end{array}$ & & & L5 & Terminal/terminal & $\mathrm{L} 4: \times / x$ & 275 \\
\hline 6 & M & 14 & Run & L4+L5 & 2 & L4 & Early/- & & & L5 & -/early & L4: $\bigcirc /-$, L5: $-/ \bigcirc$ & 98 \\
\hline 7 & M & 15 & Tennis & $\mathrm{L} 1+\mathrm{L} 2$ & 2 & L1 & Terminal/- & & & L2 & -/terminal & & \\
\hline 8 & M & 14 & Soccer & $\mathrm{L} 1+\mathrm{L} 4+\mathrm{L} 5$ & 4 & L1 & -/terminal & L4 & -/early & L5 & Terminal/terminal & L4: $-/ \bigcirc$ & 126 \\
\hline 9 & M & 14 & Speed skate & $\mathrm{L} 3+\mathrm{L} 4$ & 3 & L3 & Terminal/- & & & L4 & $\begin{array}{l}\text { Progressive/ } \\
\text { progressive }\end{array}$ & L4: $\bigcirc / \bigcirc$ & 99 \\
\hline 10 & M & 17 & Athletics & L4+L5 & 4 & L4 & Terminal/terminal & & & L5 & Terminal/terminal & L4: $x / x$ & 155 \\
\hline 11 & M & 16 & Baseball & L4+L5 & 3 & L4 & Progressive/- & & & L5 & Terminal/terminal & L4: $\bigcirc /-$ & 94 \\
\hline 12 & M & 16 & Baseball & $\mathrm{L} 2+\mathrm{L} 4$ & 2 & $\mathrm{~L} 2$ & Early/- & & & L4 & -/early & L2: $\bigcirc /-$, L4- $\bigcirc$ & 96 \\
\hline 13 & M & 16 & Baseball & $\mathrm{L} 2+\mathrm{L} 3+\mathrm{L} 5$ & 5 & L2 & Terminal/terminal & L3 & -/early & L5 & Terminal/terminal & L3: $-/ \bigcirc$ & 109 \\
\hline 14 & M & 17 & Rugby & $\mathrm{L} 2+\mathrm{L} 4$ & 4 & L2 & Early/early & & & L4 & Terminal/terminal & L2: $\bigcirc / \bigcirc$ & 148 \\
\hline 15 & $\mathrm{~F}$ & 17 & Tennis & L3+L6 & 3 & L3 & -/terminal & & & L6 & Terminal/terminal & & \\
\hline 16 & M & 16 & Athletics & L3+L4+L5 & 4 & L3 & Early/- & L4 & -/early & L5 & Terminal/terminal & L3: $\bigcirc /-$, L4: $-/ \bigcirc$ & 217 \\
\hline 17 & M & 14 & Baseball & $\mathrm{L} 3+\mathrm{L} 4+\mathrm{L} 5$ & 5 & L3 & Early/early & L4 & Early/- & L5 & Terminal/terminal & L3: $\bigcirc / \bigcirc$, L4: $\bigcirc /-$ & 82 \\
\hline 18 & M & 14 & Baseball & L4+L5 & 4 & L4 & Early/early & & & L5 & Early/progressive & L4: $\bigcirc / \bigcirc, L 5: \times / \times$ & 231 \\
\hline 19 & M & 16 & Baseball & L3+L5 & 3 & L3 & Early/- & & & L5 & Early/progressive & L3: $\bigcirc /-, x / x$ & 48 \\
\hline 20 & $\mathrm{~F}$ & 13 & Baseball & L4+L5 & 3 & L4 & -/early & & & L5 & Terminal/terminal & L4: $-/ \bigcirc$ & 88 \\
\hline 21 & $\mathrm{~F}$ & 11 & Volleyball & L4+L5 & 3 & L4 & Early/- & & & L5 & Terminal/terminal & L4: $\bigcirc /-$ & 171 \\
\hline 22 & M & 16 & Baseball & $\mathrm{L} 3+\mathrm{L} 4+\mathrm{L} 5$ & 6 & L3 & Early/early & L4 & $\begin{array}{c}\text { Progressive/ } \\
\text { progressive }\end{array}$ & L5 & Terminal/terminal & $\begin{array}{c}\text { L3: } \bigcirc / \bigcirc, \\
\text { L4: } \times / \times\end{array}$ & 93 \\
\hline 23 & $\mathrm{~F}$ & 14 & Badminton & L4+L5 & 3 & L4 & -/early & & & L5 & Terminal/terminal & L4: $-/ \bigcirc$ & 69 \\
\hline
\end{tabular}

The cases with at least more than one site classified as early or progressive stage received conservative treatment to achieve bone union. All patients stopped playing sports, received fixation with a soft brace for sports (Max belt S3 ${ }^{\circledR}$, SIGMAX), and underwent athletic rehabilitation focused on core training and stretching. The cases in the terminal stage at all spinal levels were not expected to achieve bone union, so those cases underwent athletic rehabilitation until their low back pain disappeared and they could return to playing sports.

The combination of stages was evaluated in three groups. The cases in the early or progressive stage at all spinal levels were defined as the "early or progressive+early or progressive" group, and the cases in the terminal stage at all spinal levels were defined as the "terminal+terminal" group. Then the cases in the early or progressive stage with at least one spinal level and in the terminal stage with at least one different spinal level were defined as "early or progressive+ terminal" group.

Bone union was evaluated using lumbar CT. Only the affected vertebrae were examined every 1 to 2 months until bone healing was obtained or the patient exhibited pseudarthrosis. Because bone union on the most ventral side of the vertebral arch can take a long time, we assessed bone union as being achieved when healing had occurred in at least $75 \%$ of the pars from the ventral to the dorsal side on the sagittal images. On the basis of this, we investigated the prevalence and bone union rate of multiple-level spondylolysis among the growth-stage children.

\section{Results}

A total of 782 patients were examined for LBP. Lumbar spondylolysis was diagnosed in 243 patients. Of these, 220 patients had single-level spondylolysis. Multiple-level spondylolysis was observed in 23 patients (17 men and 6 women with a mean age of $15.0 \pm 1.6$ years [13-17 years] ) at 83 sites (Table 1). The number of vertebrae with pars defect was 2 in 17 cases, 3 in 6 cases, and $\geq 4$ in 0 cases. In terms of disease stage, 28 sites were in the early stage, 11 were in the progressive stage, and 44 were in the terminal stage (Table 2). Thus, the prevalence of multiple-level spondylolysis among patients with LBP was $2.9 \%$, and the prevalence of multiple-level spondylolysis among all cases of lumbar spondylolysis was $9.5 \%$. Of the children diagnosed with multiple-level spondylolysis, most (20/23 cases, 87.0\%) had at least over one site of pars defects in the early or progressive stage in which bone union could be expected. There 
Table 2. Number of Pars Defects by Stage Classification.

\begin{tabular}{lcc}
\hline Stage classification & Number of pars defects & Percentage $(\%)$ \\
\hline Early & 28 & 33.7 \\
Progressive & 11 & 13.3 \\
Terminal & 44 & 53.0 \\
All & 83 & - \\
\hline
\end{tabular}

Table 3. Number of Cases by Combination of Stages.

\begin{tabular}{lcc}
\hline \multicolumn{1}{c}{ Combination of stages } & $\begin{array}{r}\text { Number } \\
\text { of cases }\end{array}$ & $\begin{array}{c}\text { Percentage } \\
(\%)\end{array}$ \\
\hline Early or progressive+early or progressive & 2 & 8.7 \\
Early or progressive+terminal & 18 & 78.3 \\
Terminal+terminal & 3 & 13.0 \\
All & 23 & - \\
\hline
\end{tabular}

Table 4. Bone Union Rate by Stage Classification.

\begin{tabular}{lcc}
\hline & Bony union rate (\%) & $\begin{array}{c}\text { Number of union/ } \\
\text { separation sites }\end{array}$ \\
\hline Early & 92.9 & $26 / 28$ \\
Progressive & 45.5 & $5 / 11$ \\
All & 82.1 & $28 / 39$ \\
\hline
\end{tabular}

\section{- First visit}
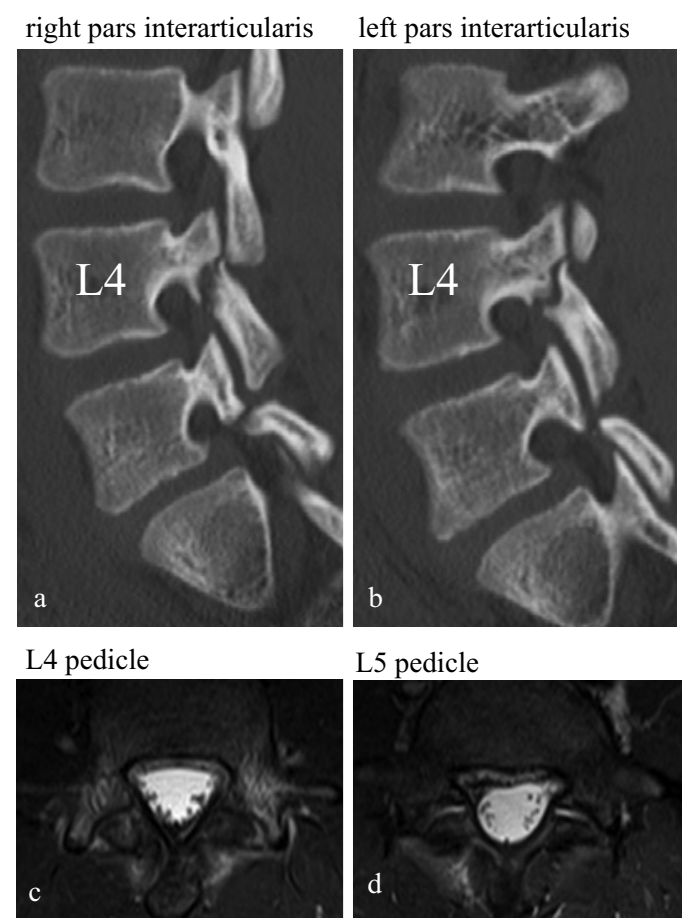

were 2 cases $(8.7 \%)$ in the "early or progressive+early or progressive" group, 18 cases $(78.3 \%)$ in the "early or progressive+terminal" group, and 3 cases $(13.0 \%)$ in the "terminal+terminal" group (Table 3).

Twenty children diagnosed with multiple-level spondylolysis who also had pars defects in the early or progressive stage received conservative treatment for bone union, which was achieved in 31 of 39 sites $(79.5 \%)$. The bone union rate by stage was $92.9 \%$ (26 of 28 sites) in the early stage and $45.5 \%$ ( 5 of 11 sites) in the progressive stage (Table 4 ). The final bone union or pseudarthrosis evaluation with CT was achieved after $126.1 \pm 57.9$ days on average after the start of treatment.

\section{Case reports}

A boy aged 14 years (Case No.5) had a chief complaint of low back pain when playing soccer. CT performed at the first visit showed defects with complete bilateral separation of the pars at L4 and L5 (Fig. 2a, b). The signal intensity at the adjacent vertebral pedicles in lumbar MRI T2FS was high for both sides of L4 (Fig. 2c) and low for both sides of L5 (Fig. 2d). The stage classifications were bilateral progressive stage at L4 and bilateral terminal stage at L5. Conservative treatment was performed to achieve bilateral bone union of L4 for approximately 9 months. On both sides, the separation defect did not expand, and osteogenesis was ob-

\section{.9 months later}

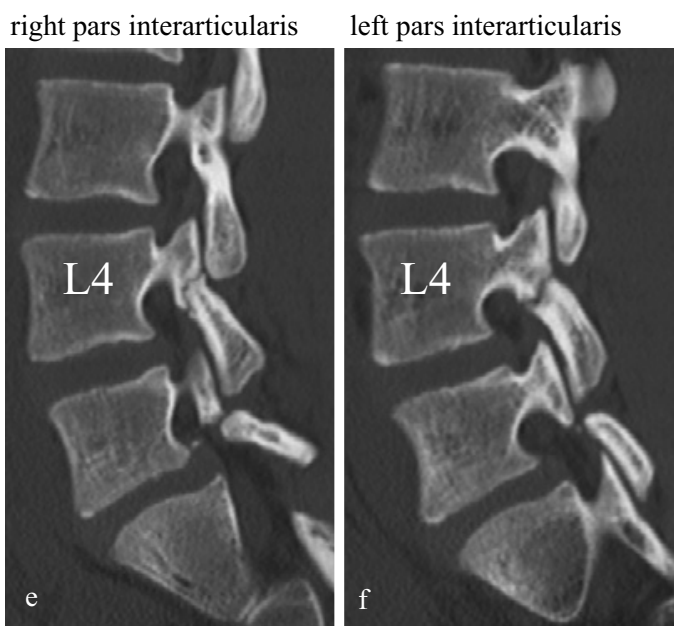

Figure 2. Images of computed tomography (CT) and magnetic resonance imaging (MRI) of the boy aged 14 years (Case No. 5).

CT sagittal plane through right and left pars interarticularis at first visit (a,b). MRI T2 fat suppressed imaging axial scan through L4 and L5 pedicles at first visit (c,d). CT sagittal plane through right and left pars interarticularis at 9 months later $(e, f)$. 
served internally, but complete bone union was not achieved, and pseudarthrosis occurred (Fig. 2e, f).

\section{Discussion}

In this study, we found lumbar spondylolysis in 243 of 782 cases $(31.1 \%$ ) among growth-stage children (age 6-18 years) with LBP. Twenty-three cases of multiple-level spondylolysis accounted for $2.9 \%$ of LBP. Further, multiple-level spondylolysis made up $9.5 \%$ of all lumbar spondylolysis cases. According to Sakai et al. ${ }^{2}$, lumbar spondylolysis with terminal stage separation was observed in 117 of 2,000 $(5.9 \%)$ people in a general Japanese population $(\geq 20$ years old). Multiple-level spondylolysis was observed in 5 cases $(0.25 \%)$. Further, multiple-level spondylolysis made up $4.3 \%$ of all lumbar spondylolysis cases. The growth-stage children with LBP in this study exhibited a higher rate of multiplelevel spondylolysis than adults. This could be because even if spondylolysis occurs at multiple levels in the growth stage, bone union is possible, which lowers this rate among adults.

Lumbar MRI is useful in the early diagnosis of lumbar spondylolysis ${ }^{5}$. Goda et al. ${ }^{9)}$ reported that MRI revealed a higher prevalence of L3 or L4 spondylolysis than observed with CT in growth-stage children. The lumbar spinal level of the pars defect was $66.5 \%$ for L5 and $33.7 \%$ for L3 or L 4. Of these, $32.4 \%$ of cases with pars defects in early or progressive stage at L3 or L4 also had these in terminal stage at L5. They reported that several cases of multiplelevel spondylolysis exhibited pars defects in early or progressive stage complicated with these in terminal stage. In this study, the combination of terminal stage at one spinal level and early or progressive stage at a different spinal level was observed in 18 cases $(78.3 \%)$. When diagnosing growth-stage children with LBP, if there were obvious pars defects in the terminal stage for spondylolysis in lumbar radiography, this may be considered as the cause of LBP; thus, further examination would not be conducted. This would suggest that multiple-level spondylolysis was likely overlooked. When a growth-stage child complains of LBP, even if they had obvious pars defects in the terminal stage in lumbar radiography, MRI or CT should also be performed because of the possibility of separation starting from the ventral side at different spinal level.

We diagnosed lumbar spondylolysis using lumbar CT and MRI and categorized lumbar spondylolysis by disease stage based on a modification of the categorizations used by Fujii et al. ${ }^{7)}$ and Sairyo et al. ${ }^{8}$. Fujii et al. categorized the stage by oblique axial CT images sliced along the pars interarticularis $^{7)}$. In the early stage, a hairline crack is visible in the pars. In the progressive stage, an obvious narrow gap is visible, but its edge is round. In the terminal stage, a wide gap is visible, and the edge is sclerosed. Subjective judgment is required, which is sometimes difficult if using only the oblique axial images. For this reason, we used sagittal CT images, which can be evaluated according to the degree of separation starting from the ventral side and extending to the dorsal side of the vertebral arch. Among the pars defects exhibiting bone marrow edema in the adjacent vertebral pedicle, the defects limited to the ventral side of the vertebral arch and bony continuity on the dorsal side were considered in the early stage, whereas the defects extending to the dorsal side and with complete separation of the vertebral arch were considered in the progressive stage. There was no significant difference in the stage between the oblique axial and sagittal images. The sagittal images were very useful when the judgment was difficult using only the oblique axial images. Sairyo et al. ${ }^{10)}$ reported that the bone union rate of the cases not exhibiting bone marrow edema in the adjacent vertebral pedicle of the pars defects on MRI was $0 \%{ }^{10}$. The presence or absence of bone marrow edema is useful in determining whether bone union is possible. When bone marrow edema was not detected on MRI, we confirmed the separations on CT images. In traditional classification ${ }^{7)}$, if the narrow gap was visible not wide gap on oblique axial CT images, the defect was considered in progressive stage. In this study, these defects which not detected bone marrow edema on MRI were all wide gap, and the edges were all sclerosed on CT oblique axial and sagittal images, and considered in terminal stage. We placed more importance on hypointense signal on $\mathrm{T} 1$ than hyperintense signal on $\mathrm{T} 2$ or T2FS when bone marrow edema was confirmed on MRI. The reason was because the signal intensity of the bone marrow edema at the adjacent vertebral pedicle of the pars defects had disappeared earlier the hyperintense signal on $\mathrm{T}$ 2 or T2FS than the hypointense signal on T1. If the hyperintense signal on T2 or T2FS had disappeared but the hypointense signal on $\mathrm{T} 1$ remained at the pedicle, we considered bone marrow edema was detected. If the hypointense signal on $\mathrm{T} 1$ had disappeared, we considered no bone marrow edema. These defects with no bone marrow edema were all wide gap and the edges were all sclerosed, we considered in terminal stage. The stage classification in this study was slightly different from the previous classification; thus, the result of bone union rates may differ from that of previous reports.

There have been no previous reports on bone union rates with conservative treatment for multiple-level spondylolysis. In this study, the bone union rate of stress fractures in multiple-level spondylolysis was $79.5 \%$. The rate was $92.9 \%$ and $45.5 \%$ in the early and progressive stages, respectively. Sairyo et al. reported that the rate was $94 \%$ and $64 \%$ in the early stage and progressive single-level spondylolysis, respectively ${ }^{8}$. Multiple-level and single-level spondylolysis exhibited similar bone union rates in the early stage. A finite element analysis by Sairyo et al. found that the pars defects in the terminal stage at L5 reduced the load on the pars interarticularis of the vertebral arch of $\mathrm{L} 4^{11)}$. This suggests that the possibility of bone union was as high in multiple-level spondylolysis as that in single-level spondylolysis. However, the bone union rate in the progressive stage cases in this study was somewhat low. This low rate seems to be because 
of the $0 \%$ bone union rate ( 0 of 6 sites) in the bilateral progressive stage at L4 combined with that in the bilateral terminal stage at L5. In single-level spondylolysis, bilateral spondylolysis in the progressive stage had lower bone union rate $^{7}$. Thus, the possibility of bone union in the bilateral progressive stage may also be low in multiple-level spondylolysis. This study had a small sample size. In the future, we need to examine more cases. Conservative treatment to achieve bone union should be aggressively performed in cases of multiple-level spondylolysis, as the bone union rates can be expected similar to the cases of single-level spondylolysis.

Conflicts of Interest: The authors declare that there are no relevant conflicts of interest.

Sources of Funding: No funds were received in support of this work. No benefits in any form have been or will be received from any commercial party related directly or indirectly to the subject of this study.

Author Contributions: The authors are those who were involved in the treatment of sports disorders and lumbar spondylolysis at related facilities.

Ethical Approval: This study was approved by the Ethics Committee of the Obihiro Kyokai Hospital (Obihiro city, Hokkaido, Japan). The approval number is "Obi-Kyokai 2015-16."

\section{References}

1. Fredrickson BE, Baker D, McHolick WJ, et al. The natural history of spondylolysis and spondylolisthesis. J Bone Joint Surg Am. 1984;66(5):699-707.
2. Sakai T, Sairyo K, Takao S, et al. Incidence of lumbar spondylolysis in the general population in Japan based on multidetector computed tomography scans from two thousand subjects. Spine. 2009; 34(21):2346-50.

3. Grantham SA, Imbriglia JE. Double-level spondylolysis and transitional vertebra. Case report. J Bone Joint Surg Am. 1975;57(5): 713-4.

4. Liu X, Wang L, Yuan S, et al. Multiple-level lumbar spondylolysis and spondylolisthesis. J Neurosurg Spine. 2015;22(3):283-7.

5. Sairyo K, Katoh S, Takata Y, et al. MRI signal changes of the pedicle as an indicator for early diagnosis of spondylolysis in children and adolescents:a clinical and biomechanical study. Spine. 2006;31(2):206-11.

6. Terai T, Sairyo K, Goel VK, et al. Spondylolysis originates in the ventral aspect of the pars interarticularis. A clinical and biomechanical study. J Bone Joint Surg Br. 2010;92(8):1123-7.

7. Fujii K, Katoh S, Sairyo K, et al. Union of defects in the pars interarticularis of the lumbar spine in children and adolescents. $\mathrm{J}$ Bone Joint Surg Br. 2004;86(2):225-31.

8. Sairyo K, Sakai T, Yasui N, et al. Conservative treatment for pediatric lumbar spondylolysis to achieve bone healing using a hard brace:what type and how long? J Neurosurg Spine. 2012;16(6): 610-4.

9. Goda Y, Toshinori S, Sakamaki T, et al. Analysis of MRI signal changes in the adjacent pedicle of adolescent patients with fresh lumbar spondylolysis. Eur Spine J. 2014;23(9):1892-5.

10. Sairyo K, Katoh S, Takata Y, et al. MRI signal changes of the pedicle as an indicator for early diagnosis of spondylolysis in children and adolescents- a clinical and biomechanical study. Spine. 2006;31(2):206-11.

11. Sairyo K, Sakai T, Yasui N, et al. Newly occurred L4 spondylolysis in the lumbar spine with pre-existence L5 spondylolysis among sports players:case reports and biomechanical analysis. Arch Orthop Trauma Surg. 2009;129(10):1433-9.

Spine Surgery and Related Research is an Open Access journal distributed under the Creative Commons Attribution-NonCommercial-NoDerivatives 4.0 International License. To view the details of this license, please visit (https://creativeco mmons.org/licenses/by-nc-nd/4.0/). 